\title{
Capacidades reprodutivas de mulheres com malformação congênita do aparelho reprodutor: Uma leitura difrativa à luz do realismo agencial de Karen Barad
}

\author{
Caynnã de Camargo Santos ${ }^{1}$ e Virgínia Ferreira ${ }^{2}$ \\ 1 Faculdade de Economia da Universidade de Coimbra, Portugal | caynnacs@gmail.com | \\ https://orcid.org/0000-0003-4069-1363 \\ ${ }^{2}$ Faculdade de Economia da Universidade de Coimbra, Portugal | virginia@fe.uc.pt | \\ https://orcid.org/0000-0003-3838-054X
}

\begin{abstract}
Resumo: Introdução: Esforços investigativos advindos da biomedicina e da sociologia têm proposto análises sobre a infertilidade feminina cujas insuficiências contribuem para a perpetuação de compreensões essencialistas acerca dos corpos e de suas propriedades. Objetivos: O estudo pretende evidenciar como a modalidade de neomaterialismo proposta pela física e feminista norte-americana Karen Barad, nomeada de realismo agencial, e o método analítico de leitura difrativa sistematizado pela autora permitem-nos edificar compreensões alternativas acerca da infertilidade feminina, apontando caminhos para a superação das insuficiências que se fazem presentes tanto em visadas biomédicas quanto sociológicas sobre o tema. Métodos: A presente pesquisa mobiliza uma abordagem metodológica qualitativa do tipo estudo de casos múltiplos, recorrendo a entrevistas semiestruturadas junto a três mulheres diagnosticadas como inférteis devido à Síndrome de Mayer-Rokitansky-Küster-Hauser (MRKH). O procedimento analítico conduzido é a leitura difrativa, mediante a qual lemos as vivências das participantes através de alguns dos posicionamentos ontológicos depreendidos por Karen Barad das teorizações de Niels Bohr sobre mecânica quântica. Resultados: Da leitura difrativa das vivências das três participantes da pesquisa à luz das teorizações baradianas emerge um entendimento acerca das capacidades reprodutivas de mulheres com MRKH que identifica tais atributos enquanto phenomena sociomateriais ontologicamente relacionais e em constante transformação, cuja complexidade tecnológica, política, econômica e social torna insustentável qualquer tipo de essencialismo biológico. Conclusões: O realismo agencial de Barad e seu método de leitura difrativa nos convidam a resistir à tentação de atribuir aos corpos características e capacidades constantes, universais e independentes dos contextos e aparatos materialdiscursivos que as produzem, figurando enquanto promissoras ferramentas teóricas e metodológicas para esforços investigativos qualitativos que, partindo das ciências sociais, se debruçam sobre as temáticas da saúde reprodutiva, do corpo e de suas propriedades.
\end{abstract}

Palavras-chave: Síndrome de Mayer-Rokitansky-Küster-Hauser; Infertilidade; Análise Qualitativa de Dados; Ontologia; Leitura Difrativa.

Reproductive Capabilities of Women with Congenital Malformation of the Reproductive System: A Diffractive Reading through Karen Barad's Agential Realism.

\begin{abstract}
Introduction: Biomedical and sociological investigative efforts have proposed analysis on female infertility whose limitations have contributed to the perpetuation of essentialist understandings about bodies and their properties. Goals: The study aims to show how the modality of new materialism proposed by the North American physicist and feminist Karen Barad, named agential realism, and the analytical method of diffractive reading systematized by the author allow us to build alternative understandings about female infertility, pointing out ways to overcome the shortcomings that are present both in biomedical and sociological approaches on the subject. Methods: The research mobilizes a qualitative methodological approach of multiple case study, using semi-structured interviews with three women diagnosed as infertile due to Mayer-Rokitansky-Küster-Hauser Syndrome (MRKH). We conduct the analytical procedure of diffractive reading, in order to read the experiences of the participants through some of the ontological positions derived by Karen Barad from Niels Bohr's theorizations about quantum mechanics. Results: From the diffractive reading of the experiences of the three participants through the light of the Baradian theoretical propositions, an alternative understanding of the reproductive capabilities of women with MRKH emerges, which identifies these bodily attributes as ontologically relational and constantly changing socio-material phenomena, whose technological, political, economic, and social complexity makes any kind of biological essentialism unsustainable. Conclusions: Barad's agential realism and her method of diffractive reading invite us to resist the temptation to attribute to bodies characteristics and capabilities that are constant, universal, and independent from the material-discursive contexts and apparatuses that produce them, appearing as promising theoretical and methodological tools for qualitative investigative efforts that, starting from the social sciences, focus on the topics of reproductive health, the body, and its properties.
\end{abstract}

Keywords: Mayer-Rokitansky-Küster-Hauser Syndrome; Infertility; Qualitative Data Analysis; Ontology; Diffractive Reading. 


\section{Introdução}

As ciências humanas e sociais têm observado no decorrer dos últimos anos a emergência de um renovado interesse teórico nas dimensões tangíveis dos corpos humanos e nãohumanos (Mukerji, 2015). Em meio ao conjunto de autoras/autores vinculadas/os a tal "virada material" em curso em campos como a sociologia e a antropologia - também referenciadas/os como as/os "neomaterialistas" -, destaca-se a física e feminista norteamericana Karen Barad. Descrita como "uma das mais influentes e importantes representantes do materialismo contemporâneo" (Lemke, 2014, p. 5), Barad edifica sua modalidade particular de neomaterialismo, nomeada de "realismo agencial", a partir da combinação de teorizações pós-estruturalistas e o quadro epistemológico desenvolvido pelo físico dinamarquês Niels Bohr. O realismo agencial de Karen Barad tem como algumas de suas características fundamentais o questionamento de separações rígidas entre matéria e discurso e a proposição de uma nova ontologia que privilegia o entrelaçamento à separabilidade entre entidades (Barad, 2007). Conjuntamente a tal quadro teórico, a autora sistematiza o método de leitura difrativa, um inovador procedimento analítico que, informado pelo fenômeno físico de difração, promove a leitura de dados e construtos teóricos "um através dos outros" (Barad, 2003, p. 803), atentando para o modo como novas compreensões e sentidos emergem dessas interferências coconstitutivas.

O presente artigo objetiva demonstrar brevemente como as propostas teóricas e metodológicas de Karen Barad oferecem promissoras ferramentas para esforços investigativos qualitativos que, partindo das ciências sociais, se debruçam sobre as temáticas do corpo e de suas propriedades. Para tanto, voltamo-nos para as vivências de mulheres acometidas por uma forma rara de malformação do aparelho reprodutor, que se manifesta na ausência congênita do útero e agenesia vaginal (Rabelo, 2018), conhecida na literatura médica como Síndrome de Mayer-Rokitansky-Küster-Hauser (MRKH), de modo a abordar especificamente as capacidades reprodutivas de seus corpos.

As ciências médicas identificam mulheres com MRKH enquanto portadoras de uma congênita "infertilidade devido a fator uterino absoluto", isto é, incapacidade de gerar filhos decorrente da ausência ou malformação do útero (Richards et al., 2019). Nesse sentido, é possível afirmar que o discurso médico concebe as capacidades reprodutivas de mulheres com MRKH em termos estritamente causais, deterministas e universais: sendo o útero um dos principais órgãos do sistema reprodutor feminino, e tendo em vista que mulheres com MRKH não têm útero (ou o têm em forma rudimentar), conclui-se que mulheres com MRKH são invariavelmente incapazes de reprodução.

Em contraposição às visadas biomédicas, interpelações sociológicas de corpos femininos que, como nos casos de mulheres como MRKH, vivenciam dificuldades reprodutivas, têm se dedicado à consideração dos modos como diferentes discursos significam a infertilidade e, nesse processo, definem formas socio-historicamente particulares de vivenciar tal condição (Ariza, 2014; Héritier, 1984). Abordagens dessa natureza, ao "focarem na intervenção produtiva das interpretações culturais e na importância do contexto" (Kirby, 2017, p. x), enfatizam as insuficiências das leituras estritamente biológicas, acusando-as de realizarem uma indevida universalização do fenômeno e incorrerem em perniciosos essencialismos biológicos.

No entanto, apesar de seus méritos antiessencialistas, tais visadas advindas das ciências sociais não parecem ser suficientemente radicais em seus esforços de desnaturalização. De fato, o construtivismo cultural que, via de regra, permeia tais aproximações sociológicas, ao situar nos sentidos socioculturais seu objeto praticamente exclusivo de escrutínio, implicitamente relega propriedades orgânicas e capacidades corporais à condição de espontaneidades biológicas preexistentes e estáveis, contrastando com o caráter construído e variável atribuído às estruturas linguísticas de inteligibilidade que as significam contextualmente. Ou seja, a infertilidade "construída" culturalmente parece reter um estatuto ontológico à parte dessa construção, que foge ao alcance crítico-analítico do cientista social construtivista. 
Vislumbramos assim um traço característico das abordagens construtivistas de inspiração pós-estruturalista que atualmente dominam os debates sociológicos sobre os corpos: sua dificuldade em discutir a materialidade corporal e a experiência encarnada.

Diante das insuficiências que marcam abordagens biomédicas e sociológicas, definimos as seguintes perguntas como norteadoras de nossos esforços investigativos: poderíamos pensar as capacidades reprodutivas dos corpos de mulheres com MRKH como algo mais que um passivo "fato biológico" ao qual anexamos sentidos? Seria possível conferir a tais capacidades "elas mesmas", e não apenas a seus sentidos, um caráter emergente e relacional?

Visando responder a tais indagações, promovemos um exercício de leitura difrativa, no qual lemos três casos de mulheres com MRKH diagnosticadas como inférteis à luz de determinados conceitos propostos por Karen Barad.

\subsection{O Realismo Agencial de Karen Barad}

Barad parte das teorizações de Niels Bohr sobre mecânica quântica, depreendendo das mesmas importantes implicações epistemológicas e ontológicas. Segundo Bohr, aquilo que se observa em qualquer experimento físico não é um objeto individualizado e detentor de propriedades inatas e não-relacionais - como preconizado pela metafísica individualista clássica -, mas sim o inextricável entrelaçamento de "aparatos de observação" e "objeto observado", o que ele chamará de phenomenon. O phenomenon, portanto, figura no quadro epistemológico bohriano como sua unidade fundamental, uma espécie de "átomo relacional" que marca a inseparabilidade de aparatos de observação e objeto observado, devendo ser concebido enquanto o verdadeiro referente objetivo de qualquer propriedade física medida (Bohr, 1949).

Karen Barad apresenta um contributo original às teses de Bohr ao afirmar que os phenomena não marcam apenas a inseparabilidade epistemológica de observador e observado; para ela, phenomena são unidades ontológicas primárias, constitutivas do real, substituindo a tradicional concepção de que o mundo é composto por objetos independentes e detentores de propriedades inerentes. No quadro realista agencial, a noção de phenomenon identifica a "inseparabilidade ontológica/entrelaçamento de agências intra-ativas" (Barad, 2007, p. 139), podendo ser caracterizada como a unidade material elementar, da qual entidades discretas e individualizadas (os antigos pontos de partida da metafísica individualista tradicional) derivam.

O neologismo baradiano "intra-ação" expressa o fundamental desafio que o realismo agencial direciona à metafísica individualista: se o conceito de interação pressupõe a existência prévia de duas ou mais entidades individualizadas que subsequentemente se relacionam, a noção de intra-ação nomeia o processo interno aos phenomena a partir do qual entidades são performativamente materializadas enquanto "coisas" diferenciadas e determinadas. Como coloca a autora, "é por meio de intra-ações agenciais específicas que os limites e as propriedades dos 'componentes' dos phenomena se tornam determinados" (Barad, 2003, p. 815). "Coisas", portanto, não preexistem às relações, mas emergem apenas em e através de intra-ações particulares, que envolvem diferentes "aparatos de produção corporal".

Nas teorizações baradianas, "aparatos" não são redutíveis a tecnologias e instrumentos laboratoriais inertes, como sustenta Bohr. Para Barad, os aparatos são as condições materiais de possibilidade de emergência de entidades e sentidos; caracterizam, portanto, as mais diversas práticas mediante as quais inteligibilidade e materialidade são iterativamente construídas (Barad, 2007).

Em síntese, o realismo agencial caracteriza um quadro teórico transdisciplinar inovador que reconhece a inseparabilidade ontológica daquilo que habitualmente é compreendido em termos de entidades apartadas e preexistentes às relações que estabelecem entre si. Neste novo quadro teórico produz-se um fundamental deslocamento: de uma análise das relações entre coisas para uma análise de "coisas" como relações. 


\section{Metodologia}

A pesquisa se vale de uma abordagem metodológica qualitativa do tipo estudo de casos múltiplos (Yin, 2005). Entretanto, os fundamentos ontológicos e epistemológicos que assentam o realismo agencial de Karen Barad tornam forçosas algumas ressalvas e qualificações acerca dos modos como estabelecemos diálogo com formas tradicionais de pensar/fazer pesquisa qualitativa.

Das habituais abordagens qualitativas, retemos a ênfase no caráter situado e relacional dos processos de produção do conhecimento, questionando assim os fundamentos positivistas de validação do saber científico e as recorrentes pretensões de transcendência, neutralidade e universalismo que os acompanham.

Por outro lado, afastamo-nos dos pressupostos ontológicos das metodologias qualitativas humanistas tradicionais legados pela metafísica individualista clássica, na medida em que entendemos que os isolamentos a priori de dimensões do mundo (material e discursivo, natural e cultural, etc.) e de entidades variadas (corpos humanos e não-humanos, dispositivos tecnológicos, etc.) invisibilizam os entrelaçamentos constitutivos daquilo que identificamos como material, corporal e real.

Ainda, como antídoto ao antropocentrismo moderno que subjaz às abordagens qualitativas tradicionais, evocamos o princípio de simetria generalizada (Latour, 1994), de modo a advogar pela necessidade de conferir igual importância de tratamento a actantes humanos e não-humanos em nossas práticas de pesquisa e análises.

Ao endossar os fundamentos do realismo agencial baradiano, nosso estudo reconhece ser necessária a operacionalização de uma perspectiva metodológica qualitativa que (1) atente à natureza relacional e dinâmica do mundo, negando os fundamentos da metafísica individualista; (2) ao negar a premissa de existência de um abismo ontológico entre sujeito e objeto, questione também separações rígidas entre discurso e matéria, teorização e pesquisa empírica, realizando-se enquanto inextricavelmente "teorização empírica" ou "empiria teórica"; (3) acentue as dimensões éticas inerentes a qualquer prática de pesquisa assente em uma visada realista agencial, identificando aquilo que Mol (2002) chama de política ontológica em funcionamento em qualquer processo de produção de conhecimento.

Em nossos esforços analíticos não buscamos "higienizar" a aparente "desordem" (Law, 2004) dos fluxos de agências complexas que marcam o phenomenon de nosso interesse, visando que este se adapte (harmoniosa, porém artificialmente) às grelhas conceituais e interpretativas tradicionais. Além disso, não objetivamos explicar ou desvendar quaisquer "realidades" pretensamente subjacentes a relações e práticas variadas que poderiam as obscurecer. Informados pela ontologia relacional baradiana, pretendemos sublinhar em nossas análises como tais "realidades" são produzidas por, e apenas adquirem existência em, práticas e relações.

\subsection{Produção de Dados e Participantes}

À guisa de métodos empíricos para produção de informações, valemo-nos de entrevistas semi-estruturadas. Segundo Manzini (1990), essa modalidade de entrevista diferencia-se das demais por permitir a emergência de informações de modo mais livre e flexível, não condicionando as respostas a uma rígida padronização de alternativas (Manzini, 1990, p. 154).

No presente texto, convocamos informações decorrentes de três entrevistas. O grupo de participantes foi composto de três mulheres com MRKH com idades entre 33 e 42 anos, sendo duas entrevistadas brasileiras e uma entrevistada portuguesa. A seleção de participantes se deu segundo técnica de bola de neve. 
Vinuto (2014) coloca que esta é uma forma de amostragem não probabilística que utiliza de cadeias de referência e é especialmente útil para estudos que envolvem grupos difíceis de serem acessados, como é o caso de pessoas afetadas pela MRKH, cuja incidência é de 1 caso em cada 4000 a 5000 nascimentos do sexo feminino (Evans, Poland, \& Boving, 1981).

As entrevistas foram realizadas via videochamadas entre os meses de Maio e Setembro de 2020. A opção pela realização das entrevistas via videochamadas se deu tendo em vista critérios de conveniência e o imperativo de distanciamento social imposto pela situação epidemiológica instaurada pela pandemia do novo coronavírus. As entrevistas foram conduzidas com base em um guião contendo perguntas abertas, que versavam sobre as diversas dimensões das vivências de mulheres com MRKH e as relações entre a Síndrome e restrições reprodutivas. As entrevistas foram gravadas (apenas áudio) e seus trechos foram posteriormente transcritos.

\subsection{Análise de Dados: A Leitura Difrativa}

Sob a égide dos imperativos de similaridade e padronização, as práticas de análise qualitativa convencionais, especialmente quando falamos dos tratamentos despendidos a informações decorrentes de entrevistas, tendem a se aproximar de tentativas de produção de ordem e regularidade, na forma de categorias que apagam as diferenças e privilegiam a identidade entre coisas aparentemente semelhantes (Jackson, 2013, p. 742). Com isso, são invisibilizadas as relacionalidades complexas e as emergências inesperadas que, segundo o realismo agencial, são o cerne dos processos de vir-a-ser dos phenomena que compõem o real.

Motivadas/os pelas insuficiências dos métodos de análise convencionais, autoras/os vinculadas/os ao neomaterialismo propuseram diferentes e criativos modos de relacionarse com dados produzidos em pesquisa (Davies, 2014; Daza \& Huckaby, 2014; Springgay \& Truman, 2018). À luz de tais contribuições, elegemos a leitura difrativa (Barad, 2007) como nosso procedimento analítico.

Donna Haraway (2018 [1997]) propõe a noção de difração enquanto uma metáfora para expressar "outra forma de consciência crítica" (Haraway, 2018, p. 273) que deveria permear investigações calcadas nos novos modelos feministas de objetividade forte, de modo a suplantar a longeva metáfora óptica da reflexão que informa os processos de produção do conhecimento científico. Segundo a autora, a tradicional prática crítica da reflexividade, à semelhança do fenômeno físico que a inspira, promove o mero espelhamento ou replicação do "mesmo" em outro lugar.

Contrastivamente, o principal interesse de uma forma difrativa de ver e pensar, segundo Haraway, é romper com a cadeia de repetição do "mesmo" ad aeternum, atentando para os processos mediante os quais diferenças são relacionalmente produzidas. Informada pelo fenômeno físico de difração - que consiste no resultado da interação que toma parte quando ondas encontram um obstáculo ou se sobrepõem umas às outras -, tal forma de pensamento busca analisar como diferenças são criadas no mundo e quais efeitos elas têm sobre sujeitos e corpos, norteada pelo imperativo ético-político de produzir "more promising interference patterns on the recording films of our lives and bodies" (Haraway, 2018, p. 16).

Karen Barad (2007) superará os limites do uso metafórico do conceito, buscando sistematizar a leitura difrativa enquanto um método de análise. Para a autora, o principal aspecto do fenômeno físico de difração a ser retido por esforços analíticos situados diz respeito ao caráter coconstitutivo do movimento realizado por ondas quando estas se sobrepõem ou alcançam uma obstrução, dando origem a novas combinações de ondas e padrões de interferência construtiva e destrutiva. 
Analogamente à forma como ondas, ao interferirem entre si ou com obstáculos, não reproduzem mecanicamente o "mesmo", mas materializam algo novo (isto é, produzem novas combinações e padrões de difração), Barad propõe o método difrativo enquanto uma prática de promoção de interferências entre contribuições de diversas áreas disciplinares e posições teóricas, visando observar como novos phenomena (novos construtos teóricos, corpos, alternativas políticas, etc.) emergem destes entrelaçamentos e, nesse processo, rompem com o círculo vicioso da repetição, próprio da reflexão (Barad, 2007).

Em nossa tentativa de ler difrativamente visões "uma através das outras" (Barad, 2003, p. 803), mobilizamos o realismo agencial baradiano enquanto uma grade de difração que, quando atravessada pelas informações decorrentes das entrevistas, faz emergir novas formas de pensar as vivências de mulheres com MRKH.

O foco de nosso modelo analítico reside em atentar para os momentos em que dados e teorias interferem entre si construtivamente, tomando como critério para aferição do nível de "construtividade" das interferências não apenas a medida em que as propostas resultantes ampliam o saber científico vigente sobre as problemáticas de interesse, mas também a intensidade com que as mesmas apontam alternativas políticas capazes de tornar vidas hoje vitimadas por estruturas sociais de poder-saber excludentes e opressivas, mais vivíveis (Butler, 2018).

Em suma, nosso esforço de leitura difrativa almeja observar não o que é entendido, mas - que é dinâmica e intra-ativamente produzido diferencialmente pelos/nos entrelaçamentos da pesquisa.

\section{Resultados e Discussão}

llustremos o funcionamento do procedimento de leitura difrativa a partir de um trecho da fala de $J$, participante portuguesa que recebeu o diagnóstico de infertilidade associada à $\mathrm{MRKH}$ aos 17 anos. À semelhança das demais entrevistadas, ela afirma que o aspecto da Síndrome que "mais Ihe custou" aceitar diz respeito às restrições reprodutivas associadas à doença. Enquanto discorre sobre sua história, ela faz uma afirmação que parece desafiar os entendimentos amplamente compartilhados acerca das capacidades reprodutivas de mulheres com MRKH:

J: Nos casos de Rokitansky - é uma coisa curiosa -, nós somos

inférteis,mas, ao mesmo tempo, não somos (Entrevista com J, 37min 38s).

Leiamos difrativamente a fala de $J$ através de um dos pilares do realismo agencial, o princípio de indeterminação ontológica. No âmbito da mecânica quântica, entes físicos podem apresentar características variáveis dependendo das circunstâncias experimentais às quais são submetidos. No clássico experimento da dupla fenda, por exemplo, elétrons lançados em direção a um anteparo com duas fendas ora apresentam "natureza" ondulatória, ora corpuscular, alternando seu estatuto de acordo com modificações feitas no aparato observacional. Assente nas teorizações de Niels Bohr, Barad aponta que tais resultados experimentais denunciam as insuficiências da metafísica individualista, que promulga a existência de entidades autônomas e detentoras de atributos intrínsecos. Para a autora, "there are no inherently bounded and propertied things that precede their intraaction with particular apparatuses" (Barad, 2012, p. 80) - isto é, a determinação da natureza de uma "entidade" depende da especificação dos aparatos mobilizados contextualmente no ato de sua "observação". Os arranjos físicos que caracterizam cada contexto experimental desempenham um papel produtivo em relação aos "objetos" que pretensamente apenas observariam a partir de uma posição de exterioridade. Segundo o princípio da indeterminação, portanto, entidades não possuem propriedades essenciais e alheias aos contextos; são os aparatos em funcionamento em cada contexto particular os responsáveis pela resolução local da indeterminação ontológica dos "objetos". 
Ao aludir a determinada ambiguidade ontológica dos corpos de mulheres com MRKH, afirmando que eles são inférteis e não $O$ são, $J$ aponta para $o$ fato de atributos e capacidades corporais serem realidades relacionais (phenomena) que assumem conformações variáveis de acordo com os contextos nos quais se inserem, não se tratando de inflexíveis essências transcendentais preexistentes. Nesse quadro, capacidades reprodutivas e o "contexto" são vistos como não estabelecendo entre si uma relação de rígida exterioridade. As primeiras estão inextricavelmente entrelaçadas ao "social" e apenas alcançam suas instanciações particulares em meio a intra-ações com agências e práticas sociomateriais que, prima facie, aparentam ser rigidamente extracorporais, como fatores de ordem tecnológica, política, econômica e cultural. Segundo o realismo agencial, tais fatores contextuais são "aparatos de produção corporal"; em semelhança aos arranjos laboratoriais no experimento da dupla fenda, consistem em agências que solucionam localmente a inerente indeterminação ontológica dos corpos com MRKH, materializandoos enquanto capazes ou incapazes de reprodução. Não especificados os aparatos constitutivos em funcionamento em cada contexto, tais corpos não podem, a rigor, ser identificados inequivocamente como férteis ou inférteis.

Tal compreensão é corroborada pelas vivências das demais entrevistadas. Por exemplo, a participante $S$, brasileira também diagnosticada como infértil devido à $\mathrm{MRKH}$, se tornou mãe de uma filha biológica mediante um procedimento de gestação de substituição, uma modalidade de reprodução medicamente assistida em que uma doadora "cede" temporariamente seu útero para gestar o filho de outra mulher que não pode levar a cabo a gravidez. Apesar da malformação uterina, a função ovariana de mulheres com MRKH é normal, tornando possível para S se valer da técnica como meio de alcançar a maternidade biológica. Nesse caso, temos o funcionamento de um conjunto amplo de aparatos (tecnologias reprodutivas como a fertilização in vitro, medicamentos variados, a ação de diversos profissionais especializados, etc.), responsáveis por performar o corpo de Scomo capaz de reprodução.

Por outro lado, $A L$, participante também brasileira, vê seu sonho de ter um filho biológico frustrado pelas barreiras econômicas que condicionam 0 acesso às dispendiosas tecnologias reprodutivas conceptivas. Em seu caso, fatores como desemprego e o acelerado empobrecimento das populações posicionadas em estratos inferiores da pirâmide socioeconômica brasileira figuram como importantes aparatos que contribuem para a produção e mantimento de sua "incapacidade reprodutiva".

\section{Conclusões}

Da leitura difrativa das vivências de mulheres com MRKH através das teorizações baradianas emerge um convite a resistirmos à tentação de atribuir aos corpos características e capacidades constantes e independentes dos contextos e aparatos material-discursivos que as produzem. O exercício analítico proposto permite-nos reconhecer que, estando os corpos sempre-já inseridos em contextos mutantes, o conjunto de seus atributos estará também em perene transformação, de acordo com as intra-ações que tomam parte em diferentes situações. Nesse sentido, tentativas de identificação de suas "essências eternas" (características que Ihes seriam comuns em todos os espaços e tempos) são vistas enquanto esforços reféns da metafísica individualista clássica e sua ilusória crença na independência ontológica dos "objetos" do mundo.

Como colocam St. Pierre, Jackson, \& Mazzei (2016), o neomaterialismo desafia-nos a repensar o modo como compreendemos o "real", assim como incita-nos a reavaliar criticamente as formas como fazemos pesquisa. Perspectivadas via o realismo agencial de Karen Barad e seu método de leitura difrativa, fertilidade e infertilidade se apresentam enquanto phenomena sociomateriais em constante transformação, cuja complexidade tecnológica, política, econômica e social torna insustentável qualquer tipo de essencialismo biológico. 


\section{Referências}

Ariza, L. (2014). La construcción narrativa de la infertilidad. Mujeres que narran la experiencia de no poder concebir. Sexualidad, Salud y Sociedad, 00(18), 41-73.

Barad, K. (2007). Meeting the Universe Halfway: Quantum Physics and the Entanglement of Matter and Meaning. Durham \& London: Duke University Press.

Barad, K. (2012). Intra-actions [Interview with Adam Kleinman]. Mousse Magazine, 34, 76-81.

Barad, K. (2003). Posthumanist Performativity: Toward an Understanding of How Matter Comes to Matter. Signs: Journal of Women in Culture and Society, 28(3), 801-31.

Bohr, N. (1949). Discussion with Einstein on Epistemological Problems in Atomic Physics. In P. A. Schilpp (Ed.), Albert Einstein: Philosopher-Scientist (pp. 201-241). Illinois: Open Court.

Butler, J. (2018). Corpos em aliança e a política das ruas: notas para uma teoria performativa da assembleia. Rio de Janeiro: Civilização Brasileira.

Davies, B. (2014). Reading anger in early childhood intra-actions: A diffractive analysis. Qualitative Inquiry, 20(6), 734-741.

Daza, S. \& Huckaby, M. F. (2014). Terra Incognita: Em-bodied data analysis. Qualitative Inquiry, 20(6), 801-810.

Evans, T. N., Poland, M. L., \& Boving, R. L. (1981). Vaginal malformations. American Journal of Obstetrics and Gynecology, 141(8), 910-920.

Foucault, M. (2000). Microfísica do poder. Rio de Janeiro: Graal.

Haraway, D. (1988). Situated knowledges: The science question in feminism and the privilege of partial perspective. Feminist Studies, 14, 575-599.

Haraway, D. (2018). Modest-Witness@Second-Millennium.FemaleMan-Meets-OncoMouse: feminism and technoscience. New York: Routledge.

Haraway, D. (2008). When Species Meet. Minneapolis: University of Minnesota Press.

Héritier, F. (1984). Sterilité, aridité, sécheresse: quelques invariants de la pensée symbolique. In M. Augé \& C. Erzlich (Eds.), Le sens du mal: anthropologie, histoire, sociologie de la maladie (pp.123-154). Paris: Ėditions des Archives Contemporaines.

Jackson, A. Y. (2013). Posthumanist data analysis of mangling practices. International Journal of Qualitative Studies in Education, 26(6), 741-748.

Kirby, V. (2017). Foreword. In V. Kirby (Ed.), What if Culture was Nature all Along? (pp. viii-xii), Edinburgh: Edinburgh University Press.

Latour, B. (1994). Jamais Fomos Modernos: ensaio de antropologia simétrica. Rio de Janeiro: Editora 34.

Law, J. (2004). After method: Mess in social science research. Abingdon: Routledge.

Lemke, T. (2014). New Materialisms: Foucault and the 'Government of Things'. Theory, Culture and Society, 32(4), 3-25.

Manzini, E. J. (1990). A entrevista na pesquisa social. Didática, 00(26/27), 149-158.

Mol, A. (2002). The Body Multiple: Ontology in Medical Practice. Durham \& London: Duke University Press.

Mukerji, C. (2015). The Material Turn. In R. A. Scott \& S. M. Kosslyn (Eds.), Emerging Trends in the Social and Behavioral Sciences (pp.1-13). Hoboken: John Wiley \& Sons.

Rabelo, D. A. (2018). A Construção do Estigma para mulheres com Síndrome de MayerRokitansky-Küster-Hauser na Mídia - Perspectiva de Corpo. Dissertação de Mestrado, Programa de Pós-graduação em Bioética, Faculdade de Ciências da Saúde, Universidade de Brasília. 
Vol. 8 | Investigação Qualitativa em Saúde: Avanços e Desafios

Richards, E. G., Agatisa, P. K., Davis, A. C., Flyckt, R., Mabel, H., Falcone, T., Tzakis, A., \& Farrell, R. M. (2019). Framing the diagnosis and treatment of absolute uterine factor infertility: Insights from in-depth interviews with uterus transplant trial participants. AJOB Empirical Bioethics, 10(1), 23-35.

Springgay, S. \& Truman, S. (2018). Walking methodologies in a more-than-human world: WalkingLab. New York: Routledge.

St. Pierre, E. A., Jackson, A. Y., \& Mazzei, L. A. (2016). New Empiricisms and New Materialisms. Cultural Studies $\leftrightarrow$ Critical Methodologies, 16(2), 99-110.

Vinuto, J. (2014). Amostragem em bola de neve na pesquisa qualitativa: um debate em aberto. Temáticas, 22(44), 203-220.

Yin, R. K. (2005). Estudo de caso: planejamento e métodos. Porto Alegre: Bookman. 